\title{
Maximal correlation and monotonicity of free entropy and of Stein discrepancy
}

\author{
Benjamin Dadoun* $\quad$ Pierre Youssef ${ }^{\dagger}$
}

\begin{abstract}
We introduce the maximal correlation coefficient $R\left(M_{1}, M_{2}\right)$ between two noncommutative probability subspaces $M_{1}$ and $M_{2}$ and show that the maximal correlation coefficient between the sub-algebras generated by $s_{n}:=x_{1}+\ldots+x_{n}$ and $s_{m}:=x_{1}+\ldots+x_{m}$ equals $\sqrt{m / n}$ for $m \leq n$, where $\left(x_{i}\right)_{i \in \mathbb{N}}$ is a sequence of free and identically distributed noncommutative random variables. This is the free-probability analogue of a result by Dembo-Kagan-Shepp in classical probability. As an application, we use this estimate to provide another simple proof of the monotonicity of the free entropy and free Fisher information in the free central limit theorem. Moreover, we prove that the free Stein Discrepancy introduced by Fathi and Nelson is non-increasing along the free central limit theorem.
\end{abstract}

Keywords: maximal correlation; monotonicity; free entropy; free Stein discrepancy. MSC2020 subject classifications: $46 \mathrm{~L} 54 ; 62 \mathrm{H} 20$.

Submitted to ECP on November 7, 2020, final version accepted on April 6, 2021.

Supersedes arXiv: 2011.03045.

\section{Introduction and main result}

Pearson's correlation coefficient $\rho\left(X_{1}, X_{2}\right):=\operatorname{cov}\left(X_{1}, X_{2}\right) / \sigma\left(X_{1}\right) \sigma\left(X_{2}\right)$ is a standard measure of (bivariate) dependency. The maximal correlation $R\left(X_{1}, X_{2}\right)$ between two random variables $X_{1}$ and $X_{2}$ is then naturally defined as the supremum of $\rho\left(f\left(X_{1}\right), g\left(X_{2}\right)\right)$ over $\mathrm{L}^{2}$-functions $f, g$.

In 2001, Dembo, Kagan, and Shepp [7] investigated this maximal correlation for the partial sums $S_{n}:=X_{1}+\cdots+X_{n}$ of i.i.d. random variables $X_{i}$. Namely, regardless of the $X_{i}$ 's distribution, they showed that

$$
R\left(S_{n}, S_{m}\right) \leq \sqrt{\frac{m}{n}}, \quad m \leq n,
$$

with equality if $\sigma\left(X_{1}\right)<\infty$.

The aim of this note is to prove the analogous statement in the context of free probability, a theory which was initiated by Voiculescu (see [17]) in the 1980's and has since flourished into an established area with connections to several other fields. We

\footnotetext{
${ }^{*}$ Mathematics, Division of Science, New York University Abu Dhabi, UAE

E-mail: benjamin.dadoun@gmail.com

${ }^{\dagger}$ Mathematics, Division of Science, New York University Abu Dhabi, UAE.

E-mail: yp27@nyu.edu
} 
refer to $[10,12]$ for an introduction on the subject and an extensive list of references. Before stating our main result, let us first recall the necessary framework.

Let $(M, \tau)$ be a noncommutative, faithful, tracial probability space, that is a unital *-algebra $M$ equipped with a $*$-linear form $\tau: M \rightarrow \mathbb{C}$ where $\tau(1)=1$ and for all $x, y \in M$, $\tau(x y)=\tau(y x)$ (trace property), $\tau\left(x^{*} x\right) \geq 0$ (non-negativity), and $\tau\left(x^{*} x\right)=0$ if and only if $x=0$ (faithfulness). Elements of $M$ are called noncommutative random variables, and the law of $\left(x_{1}, \ldots, x_{n}\right) \in M^{n}$ is the family $\left(\tau\left(x_{i_{1}} \cdots x_{i_{r}}\right): r \geq 1,1 \leq i_{1}, \ldots, i_{r} \leq n\right)$. Finally, we recall the notion of freeness, which is the counterpart of classical independence: we say that (unital) sub-algebras $M_{1}, M_{2}, \ldots \subseteq M$ are free if "any alternating product of centered elements is centered", i.e., $\tau\left(x_{1} \cdots x_{r}\right)=0$ whenever $\tau\left(x_{j}\right)=0$ for all $1 \leq j \leq r$ and $x_{j} \in M_{i_{j}}$ with $i_{1} \neq i_{2}, i_{2} \neq i_{3}, \ldots, i_{r-1} \neq i_{r}$. In this vein, noncommutative random variables are free if the respective sub- $*$-algebras they span are free.

The inner product

$$
\langle x, y\rangle:=\tau\left(x^{*} y\right), \quad(x, y) \in M^{2},
$$

confers on $M$ a natural $\mathrm{L}^{2}$-structure with norm $\|x\|_{2}:=\sqrt{\langle x, x\rangle}$, so we may w.l.o.g. consider instead its Hilbert space completion $\mathrm{L}^{2}(M)$. More generally, $\mathrm{L}^{2}\left(M^{\prime}\right)$ will denote the closure in $\mathrm{L}^{2}(M)$ of the sub-algebra $M^{\prime} \subseteq M$. If $M^{\prime}=\mathbb{C}\left\langle x_{1}, \ldots, x_{n}\right\rangle$ is the sub- $*-$ algebra of noncommutative polynomials in $x_{1}, \ldots, x_{n} \in M$ and $x_{1}^{*}, \ldots, x_{n}^{*} \in M$, we refer to $\mathrm{L}^{2}\left(M^{\prime}\right)$ as $\mathrm{L}^{2}\left(x_{1}, \ldots, x_{n}\right)$.

Let $\operatorname{cov}(x, y):=\langle x-\tau(x) \cdot 1, y-\tau(y) \cdot 1\rangle$ and $\sigma(x)^{2}:=\operatorname{cov}(x, x)$. Just like in classical probability, we can define the Pearson correlation coefficient between $x$ and $y$ by

$$
\rho(x, y):=\frac{\operatorname{cov}(x, y)}{\sigma(x) \sigma(y)} .
$$

Note that we have $|\rho(x, y)| \leq 1$ by the Cauchy-Schwarz inequality. Given $M_{1}, M_{2} \subseteq M$ two subspaces, we call

$$
R\left(M_{1}, M_{2}\right):=\sup _{M_{1} \times M_{2}} \rho
$$

the maximal correlation coefficient between $M_{1}$ and $M_{2}$. For $M_{1}=\mathrm{L}^{2}(x), M_{2}=\mathrm{L}^{2}(y)$, we simply write $R(x, y)$, and we call it the maximum correlation between the noncommutative random variables $x$ and $y$.

We are now ready to state our main result.

Theorem 1.1. Let $\left(x_{i}\right)_{i \in \mathbb{N}}$ be a sequence of free, non-zero, identically distributed, noncommutative random variables, and let $s_{n}:=x_{1}+\cdots+x_{n}$. Then for any $m \leq n$, we have

$$
R\left(s_{n}, s_{m}\right)=\sqrt{\frac{m}{n}} .
$$

As in the classical setting, the interesting feature of the above statement is its universality as it holds regardless of the distribution of the noncommutative random variables. A possible way to prove the above statement consists of using the microstate approach by approximating the law of each noncommutative random variable by that of random matrices. One then exploits the multidimensional version of the classical maximal correlation inequality to apply it for the corresponding random matrices (seen as vectors) before passing to the limit and deducing the above theorem. The drawback of this approach is that it won't allow the extension of Theorem 1.1 to the multidimensional case. Indeed, by the refutation of Connes embedding problem [11], there are noncommutative random variables whose joint moments cannot be approximated well by moments of matrices. This makes it impossible to use the mentioned approach to prove a multidimensional version of the above theorem. On the contrast, our proof of Theorem 1.1, which is carried in Section 2, adapts the approach of [7] to a noncommutative setting and is readily extendable to the multidimensional setting. 
A celebrated result of Artstein et al [1] provided a solution to Shannon's problem regarding the monotonicity of entropy in the classical central limit theorem. In the context of free probability, the concept of free entropy and information was developped by Voiculescu in a series of papers (see for example [20]). Two approaches were given for the definition of free entropy, referred to as microstates and non-microstates and denoted by $\chi$ and $\chi^{*}$ respectively (see [12, Chapters 7 and 8 ]). These two coincide in the one-dimensional setting, in which case the free entropy of a compactly supported probability measure $\mu$ is given by

$$
\chi(\mu)=\chi^{*}(\mu):=\iint_{\mathbb{R}^{2}} \log |a-b| \mu(\mathrm{d} a) \mu(\mathrm{d} b)+\frac{3}{4}+\frac{1}{2} \log (2 \pi) .
$$

It is not known whether $\chi$ and $\chi^{*}$ coincide in the multidimensional setting. Our proof of the result presented in the sequel extends to the multidimensional case for $\chi^{*}$ only. Given a noncommutative probability space $(M, \tau)$ and a self-adjoint element $z \in M$, we define $\chi^{*}(z)$ as $\chi^{*}\left(\mu_{z}\right)$ where $\mu_{z}$ denotes the distribution of $z$, i.e., the probability measure characterized by $\int p \mathrm{~d} \mu_{z}=\tau(p(z))$ for all polynomials $p \in \mathbb{C}[X]$.

In [15], Shlyakhtenko proved the monotonicity of the free entropy in the free central limit theorem providing an analogue of the result of [1] in the noncommutative setting. As an application of our maximal correlation estimate, we recover the monotonicity property which we state in the next corollary.

Corollary 1.2. Given $\left(x_{i}\right)_{i \in \mathbb{N}}$ a sequence of free, identically distributed, self-adjoint random variables in $(M, \tau)$, one has

$$
\chi^{*}\left(\frac{s_{m}}{\sqrt{m}}\right) \leq \chi^{*}\left(\frac{s_{n}}{\sqrt{n}}\right)
$$

for every integers $m \leq n$.

As in the classical setting, the monotonicity of the entropy follows from that of the Fisher information. In Section 3, we prove the latter as a consequence of Theorem 1.1. The idea of using the maximal correlation inequality in this context goes back to Courtade [5] who used the result of Dembo, Kagan, and Shepp [7] to provide an alternative proof of the monotonicity of entropy in the classical setting.

Formulated alternatively, the above corollary states that given a compactly supported probability measure $\mu$ and positive integers $m \leq n$, one has

$$
\chi^{*}\left(m^{-1 / 2} * \mu^{\boxplus m}\right) \leq \chi^{*}\left(n^{-1 / 2} * \mu^{\boxplus n}\right),
$$

where $\boxplus$ denotes the free convolution operation (so $\mu^{\boxplus n}$ is the distribution of the sum of $n$ free copies of an element $x$ with distribution $\mu$ ), and $\alpha_{*}$ is the pushforward operation by the dilation $t \mapsto \alpha t$. As a matter of fact, it is possible to make sense of $\mu^{\boxplus t}$ for all real $t \geq 1$ (see [14]). Very recently, Shlyakhtenko and Tao [16] extended (1.2) to real-valued exponents while providing two different proofs. It would be interesting to see if the argument in this paper based on maximal correlation could be extended to cover non-integer exponents.

Another consequence of Theorem 1.1 concerns the monotonicity of the free Stein discrepancy along the free central limit theorem. Stein discrepancy measures in some sense how far is a probability measure from another one characterized by some integration by parts formula. Using the classical maximal correlation inequality, it was proven by Courtade, Fathi and Pananjady [6] that the Stein discrepancy (relative to the standard Gaussian measure) is non-increasing in the central limit theorem. The notion of free Stein discrepancy relative to a semicircular law was introduced by Fathi and Nelson [9]. Recall that a standard semicircular variable $S$ is a self-adjoint element of $(M, \tau)$ whose 
distribution has density $\frac{1}{2 \pi} \sqrt{4-t^{2}} \mathbf{1}_{[-2,2]}(t)$. Analogously to the normal distribution, a standard semicircular variable $S \in M$ is characterized by the following integration by parts formula stating that

$$
\langle S, P(S)\rangle=\langle 1 \otimes 1, \partial P(S)\rangle,
$$

for every polynomial $P$. Here, $\partial$ denotes the noncommutative derivative and the right hand side dot product refers to the dot product in the Hilbert space $\mathrm{L}^{2}(M) \otimes \mathrm{L}^{2}(M)$ (see [12]). Following [9], a free Stein kernel of $x \in M$ is an element $K \in \mathrm{L}^{2}(M) \otimes \mathrm{L}^{2}(M)$ such that

$$
\langle x, P(x)\rangle=\langle K, \partial P(x)\rangle,
$$

for every polynomial $P$. It was shown by Cébron, Fathi and Mai [4] that free Stein kernel always exist if $\tau(x)=0$. The free Stein discrepancy of $x$ relative to $S$ is then defined as

$$
\Sigma^{*}(x \mid S)=\inf _{K}\|K-1 \otimes 1\|_{\mathrm{L}^{2}(M) \otimes \mathrm{L}^{2}(M)},
$$

where the infimum is taken over all free Stein kernels $K$ of $x$. We should note that [9] introduced the notion of free Stein kernel/discrepancy relative to a general potential while we will only be dealing with the particular case of the potential $t^{2} / 2$ leading to the notions stated above.

As an application of our maximal correlation inequality, we obtain the following corollary extending the aforementioned monotonicity of Stein discrepancy obtained in [6] to the free setting.

Corollary 1.3. Given $\left(x_{i}\right)_{i \in \mathbb{N}}$ a sequence of free, centered, identically distributed, selfadjoint random variables in $(M, \tau)$ with unit norm, one has

$$
\Sigma^{*}\left(\frac{s_{n}}{\sqrt{n}} \mid S\right) \leq \sqrt{\frac{m}{n}} \Sigma^{*}\left(\frac{s_{m}}{\sqrt{m}} \mid S\right),
$$

for every integers $m \leq n$.

Note that taking $m=1$ in the above corollary, we obtain that $\Sigma^{*}\left(n^{-\frac{1}{2}} s_{n} \mid S\right)$ decays faster than $C / \sqrt{n}$ for some constant $C$ recovering a result of [4]. Similarly to the previous results of this paper, the proof of the above corollary works verbatim in the multidimensional setting.

\section{Proof of Theorem 1.1}

Let us fix $x_{1}, x_{2}, \ldots \in M$. For every finite set $I \subset \mathbb{N}$, we denote

$$
\mathrm{L}_{I}^{2}:=\mathrm{L}^{2}\left(x_{i}: i \in I\right) \quad \text { and } \operatorname{proj}_{I}(z):=\operatorname{proj}_{\mathrm{L}_{I}^{2}}(z)
$$

the orthogonal projection of $z \in M$ onto $\mathrm{L}_{I}^{2}$, which is nothing else but the conditional expectation of $z$ given $\mathrm{L}_{I}^{2}$ :

$$
y=\operatorname{proj}_{I}(z) \Longleftrightarrow y \in \mathrm{L}_{I}^{2} \text { and } \forall x \in \mathrm{L}_{I}^{2}, \tau(x y)=\tau(x z) .
$$

In particular $\operatorname{proj}_{I}(z)=z$ if $z \in \mathrm{L}_{I}^{2}$, and by the trace property

$$
\operatorname{proj}_{I}(x z y)=x \operatorname{proj}_{I}(z) y \quad \text { for all } x, y \in M \cap \mathrm{L}_{I}^{2}, z \in M .
$$

Note that $\operatorname{proj}_{\emptyset}(z)=\tau(z) \cdot 1$ and $\operatorname{proj}_{J} \circ \operatorname{proj}_{I}=\operatorname{proj}_{J}$ for every $J \subseteq I$ (tower property). When freeness is further involved, we can say a bit more. The following lemma appears in different forms in the literature ([2], [12, Section 2.5]), we include its proof for completeness. 
Maximal correlation and monotonicity of free entropy and of Stein discrepancy

Lemma 2.1. Let $I, J \subset \mathbb{N}$ be finite sets and suppose $\left(x_{k}: k \in I \cup J\right)$ is free. Then:

(i) if $z$ is a (noncommutative) polynomial in variables in $\mathbb{C}\left\langle x_{i}: i \in I\right\rangle$ and in variables in $\mathbb{C}\left\langle x_{j}: j \in J \backslash I\right\rangle$, then $\operatorname{proj}_{I}(z)$ is a polynomial in only those variables in $\mathbb{C}\left\langle x_{i}: i \in I\right\rangle ;$

(ii) the projections commute: $\operatorname{proj}_{I} \circ \operatorname{proj}_{J}=\operatorname{proj}_{I \cap J}$.

Proof. (i) By linearity of $\operatorname{proj}_{I}$, we may suppose without loss of generality that $z=$ $a_{1} \cdots a_{r}$ with each $a_{k}$ in $\mathbb{C}\left\langle x_{i}: i \in I\right\rangle$ or $\mathbb{C}\left\langle x_{j}: j \in J \backslash I\right\rangle$. From the moment-cumulant formula [12, Definition 9.2.7] w.r.t. the conditional expectation $\operatorname{proj}_{I}$, we can write

$$
\operatorname{proj}_{I}(z):=\sum_{\pi \in \mathrm{NC}(r)} \kappa_{\pi}^{I}\left(a_{1}, \ldots, a_{r}\right)
$$

where the summation ranges over all non-crossing partitions $\pi \in \mathrm{NC}(r)$ of $\{1, \ldots, r\}$ and the $\kappa_{\pi}^{I}$ are nestings (consistently with the blocks of $\pi$ ) of the free (conditional) cumulants $\kappa_{n}^{I}: M^{n} \rightarrow \mathrm{L}_{I}^{2}$ (which can be defined inductively). For instance, if

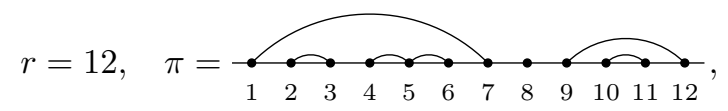

then

$$
\kappa_{\pi}^{I}\left(a_{1}, \ldots, a_{r}\right)=\kappa_{2}^{I}\left(a_{1} \cdot \kappa_{2}^{I}\left(a_{2}, a_{3}\right) \cdot \kappa_{3}^{I}\left(a_{4}, a_{5}, a_{6}\right), a_{7}\right) \cdot \kappa_{1}^{I}\left(a_{8}\right) \cdot \kappa_{2}^{I}\left(a_{9} \cdot \kappa_{2}^{I}\left(a_{10}, a_{11}\right), a_{12}\right) .
$$

As the bimodularity property (2.1) extends to conditional cumulants [12, Remark 9.1)], each of the innermost cumulants $\kappa_{s}^{I}$ is in the form

$$
b_{0} \cdot \kappa_{s}^{I}\left(n_{1} \cdot b_{1}, n_{2} \cdot b_{2}, \ldots, n_{s}\right) \cdot b_{s},
$$

where $s \in \mathbb{N}$ is some integer and the $b_{i}$ 's (resp. $n_{j}$ 's) are (possibly constant) polynomials in the subset of the variables $a_{1}, \ldots, a_{r}$ that belong to $\mathbb{C}\left\langle x_{i}: i \in I\right\rangle\left(\operatorname{resp} . \mathbb{C}\left\langle x_{j}: j \in J \backslash I\right\rangle\right.$ ). We now invoke [13, Theorem 3.6] with $F:=\left.\tau\right|_{\mathrm{L}_{I}^{2}}$ and the sub-algebra $N:=\mathbb{C}\left\langle x_{j}: j \in J \backslash I\right\rangle$ free from $B:=\mathrm{L}_{I}^{2}$ over $D:=\mathbb{C} \cdot 1$ to see that the $\kappa_{s}^{I}$ term reduces to a constant:

$$
\kappa_{s}^{I}\left(n_{1} \cdot b_{1}, n_{2} \cdot b_{2}, \ldots, n_{s}\right)=\tau\left(b_{1}\right) \cdots \tau\left(b_{s-1}\right) \cdot \kappa_{s}\left(n_{1}, n_{2}, \ldots, n_{s}\right) \in \mathbb{C}
$$

where $\kappa_{s}$ is the (unconditionned) cumulant $M^{s} \rightarrow \mathbb{C}$. By multilinearity of the cumulants, the outer nestings of $\kappa_{\text {. }}^{I}$ remain in the same form, and reducing them successively thus shows that each term $\kappa_{\pi}^{I}\left(a_{1}, \ldots, a_{r}\right)$ is in fact a polynomial only in the subset of the variables $a_{1}, \ldots, a_{r}$ that belong to $\mathbb{C}\left\langle x_{i}: i \in I\right\rangle$. This proves the claim.

(ii) Clearly $\tau\left(x \operatorname{proj}_{I} \circ \operatorname{proj}_{J}(z)\right)=\tau\left(\operatorname{proj}_{I}\left(\operatorname{proj}_{J}(x z)\right)\right)=\tau(x z)$ for all $x \in \mathrm{L}_{I \cap J}^{2}$, so we only need to check that $\operatorname{proj}_{I}\left(\operatorname{proj}_{J}(z)\right) \in \mathrm{L}_{I \cap J}^{2}$. By a closure argument, we may suppose that $\operatorname{proj}_{J}(z)$ belongs to $\mathbb{C}\left\langle x_{j}: j \in J\right\rangle$. In this $\operatorname{case}_{\operatorname{proj}_{I}}\left(\operatorname{proj}_{J}(z)\right) \in$ $\mathbb{C}\left\langle x_{k}: k \in I \cap J\right\rangle \subseteq \mathrm{L}_{I \cap J}^{2}$ by the previous point.

We now set, for every $z \in M$,

$$
z_{I}:=\sum_{J \subseteq I}(-1)^{|I|-|J|} \operatorname{proj}_{J}(z) \in \mathrm{L}_{I}^{2}
$$

where $|\cdot|$ is the cardinal notation. The following Efron-Stein (a.k.a. ANOVA) decomposition will play a crucial role in the proof of Theorem 1.1. Its use in free probability seems new (to the best of the authors' knowledge) and may be of other independent interest. 
Maximal correlation and monotonicity of free entropy and of Stein discrepancy

Lemma 2.2. For every finite set $I \subset \mathbb{N}$,

$$
\operatorname{proj}_{I}(z)=\sum_{J \subseteq I} z_{J}
$$

Proof. We repeat in a compact way the argument of Efron and Stein [8]:

$$
\begin{aligned}
\sum_{J \subseteq I} z_{J} & =\sum_{J \subseteq I} \sum_{K \subseteq J}(-1)^{|J|-|K|} \operatorname{proj}_{K}(z) \\
& =\sum_{K \subseteq I} \underbrace{\left(\sum_{K \subseteq J \subseteq I}(-1)^{|J|-|K|}\right)}_{=(1-1)^{|I \backslash K|}} \operatorname{proj}_{K}(z) \\
& =\operatorname{proj}_{I}(z) .
\end{aligned}
$$

The elements $z_{J}$ will be orthogonal thanks to this direct consequence of Lemma 2.1: Lemma 2.3. Suppose that $x_{1}, x_{2}, \ldots$ are free, and let $I, J \subset \mathbb{N}$ be finite sets such that $I \backslash J \neq \emptyset$. Then $\operatorname{proj}_{J}\left(z_{I}\right)=0$ for every $z \in M$. In particular, $z_{I}$ is orthogonal to $z_{J} \in \mathrm{L}_{J}^{2}$.

Proof. Apply Lemma 2.1 and gather the subsets $K \subseteq I$ that have same intersection $L:=J \cap K$ with $J$ :

$$
\begin{aligned}
\operatorname{proj}_{J}\left(z_{I}\right) & =\sum_{K \subseteq I}(-1)^{|I|-|K|} \operatorname{proj}_{J} \circ \operatorname{proj}_{K}(z) \\
& =\sum_{L \subseteq I \cap J}(-1)^{|I|-|L|} \underbrace{\left(\sum_{K \subseteq I \backslash J}(-1)^{|K|}\right)}_{=(1-1)^{|I \backslash J|}} \operatorname{proj}_{L}(z) \\
& =0 .
\end{aligned}
$$

To prove Theorem 1.1 we shall finally exploit that the partial sum $s_{n}:=x_{1}+\cdots+x_{n}$ is symmetric in $\left(x_{1}, \ldots, x_{n}\right)$. Our next proposition is tailored for this purpose.

Proposition 2.4. Suppose that $x_{1}, \ldots, x_{n}$ are free and identically distributed. Then for every symmetric polynomial $z=p\left(x_{1}, \ldots, x_{n}\right)$ in $x_{1}, \ldots, x_{n}$ and every $I \subseteq\{1, \ldots, n\}$, the pair $\left(z_{I}, z_{I}^{*}\right)$ has the same distribution as $\left(z_{I^{\prime}}, z_{I^{\prime}}^{*}\right)$ where $I^{\prime}:=\{1, \ldots,|I|\}$.

Consequently, if $\tau(z)=0$, then

$$
\left\|\operatorname{proj}_{I}(z)\right\|_{2} \leq \sqrt{\frac{|I|}{n}}\|z\|_{2} .
$$

Proof. Let $m=|I|$ and let $\varsigma$ be a permutation of $\{1, \ldots, n\}$ mapping $I^{\prime}$ to $I$. Since $p$ is symmetric, we can write $z=p\left(x_{1}, \ldots, x_{n}\right)=p\left(x_{\varsigma(1)}, \ldots, x_{\varsigma(n)}\right)$. By Lemma 2.1, we see from its definition in (2.2) that $z_{I}$ is a certain polynomial $q$, in the $x_{i}, i \in I$, that is

$$
z_{I}=z_{\varsigma\left(I^{\prime}\right)}=q\left(x_{\varsigma(1)}, \ldots, x_{\varsigma(m)}\right)
$$

Now the $x_{i}$ 's are free and identically distributed, so the relabelling shows that

$$
z_{I^{\prime}}=q\left(z_{1}, \ldots, z_{m}\right)
$$

with the same polynomial $q$, and thus the pairs

$$
\left(z_{I}, z_{I}^{*}\right)=\left(q\left(x_{\varsigma(1)}, \ldots, x_{\varsigma(m)}\right), q\left(x_{\varsigma(1)}, \ldots, x_{\varsigma(m)}\right)^{*}\right)
$$


Maximal correlation and monotonicity of free entropy and of Stein discrepancy

and

$$
\left(z_{I^{\prime}}, z_{I^{\prime}}^{*}\right)=\left(q\left(x_{1}, \ldots, x_{m}\right), q\left(x_{1}, \ldots, x_{m}\right)^{*}\right)
$$

have the same moments. In particular $\left\|z_{I}\right\|_{2}=\left\|z_{I^{\prime}}\right\|_{2}$, and the stated inequality becomes clear by combining this with Lemmas 2.2 and 2.3:

$$
\begin{aligned}
\left\|\operatorname{proj}_{I}(z)\right\|_{2}^{2} & =\sum_{J \subseteq I}\left\|z_{J}\right\|_{2}^{2} \\
& =\sum_{k=1}^{m}\left(\begin{array}{c}
m \\
k
\end{array}\right)\left\|z_{\{1, \ldots, k\}}\right\|_{2}^{2} \\
& \leq \frac{m}{n} \sum_{k=1}^{n}\left(\begin{array}{l}
n \\
k
\end{array}\right)\left\|z_{\{1, \ldots, k\}}\right\|_{2}^{2} \\
& =\frac{m}{n}\left\|\operatorname{proj}_{\{1, \ldots, n\}}(z)\right\|_{2}^{2} \\
& =\frac{m}{n}\|z\|_{2}^{2},
\end{aligned}
$$

using that $z_{\emptyset}=0$, that $\left(\begin{array}{c}m \\ k\end{array}\right) \leq \frac{m}{n}\left(\begin{array}{l}n \\ k\end{array}\right)$ for all $1 \leq k \leq m \leq n$, and that $z \in \mathrm{L}^{2}\left(x_{1}, \ldots, x_{n}\right)$.

Proof of Theorem 1.1. The lower bound $R\left(s_{n}, s_{m}\right) \geq \sqrt{m / n}$ is straightforward since, by freeness, $\sigma\left(s_{n}\right)^{2}=n \sigma\left(x_{1}\right)^{2}$ and $\operatorname{cov}\left(s_{n}, s_{m}\right)=\sigma\left(s_{m}\right)^{2}=m \sigma\left(x_{1}\right)^{2}$. For the upper bound, we must show that $\rho\left(z, z^{\prime}\right) \leq \sqrt{m / n}$ for all $z \in \mathrm{L}^{2}\left(s_{n}\right)$ and $z^{\prime} \in \mathrm{L}^{2}\left(s_{m}\right)$. W.l.o.g., we may suppose that $\tau(z)=\tau\left(z^{\prime}\right)=0$ and, by another closure argument, that $z$ is a polynomial in $s_{n}$ (and thus a symmetric polynomial in $x_{1}, \ldots, x_{n}$ ). Then by the Cauchy-Schwarz inequality and Proposition 2.4,

$$
\begin{aligned}
\operatorname{cov}\left(z, z^{\prime}\right) & =\left\langle z, z^{\prime}\right\rangle \\
& =\left\langle\operatorname{proj}_{\{1, \ldots, m\}}(z), z^{\prime}\right\rangle \\
& \leq\left\|\operatorname{proj}_{\{1, \ldots, m\}}(z)\right\|_{2}\left\|z^{\prime}\right\|_{2} \\
& \leq \sqrt{\frac{m}{n}}\|z\|_{2}\left\|z^{\prime}\right\|_{2} \\
& =\sqrt{\frac{m}{n}} \sigma(z) \sigma\left(z^{\prime}\right),
\end{aligned}
$$

and the proof is complete.

\section{Monotonicity of the free entropy and free Fisher information}

The goal of this section is to prove Corollary 1.2. Let us start by noting that the free entropy and free Fisher information of a self-adjoint element $z \in M$ are related through the integral formula ([18]; see also [12, Chapter 8])

$$
\chi^{*}(z)=\frac{1}{2} \int_{0}^{\infty}\left(\frac{1}{1+t}-\Phi(z+\sqrt{t} S)\right) \mathrm{d} t+\frac{1}{2} \log (2 \pi \mathrm{e}),
$$

where $S$ stands for a standard semicircular variable free from $z$, and $\Phi$ denotes the free Fisher information. After [18], the free Fisher information of a noncommutative, selfadjoint random variable $z \in M$ is defined as $\Phi(z):=\|\xi\|_{2}^{2}$ where the so called conjugate variable $\xi:=\xi(z)$ is any element of $\mathrm{L}^{2}(z)$ such that, for every integer $r \geq 0$,

$$
\tau\left(\xi z^{r}\right)=\sum_{k=0}^{r-1} \tau\left(z^{k}\right) \tau\left(z^{r-1-k}\right) .
$$


(If such a $\xi$ does not exist, we set $\Phi(z):=\infty$.) We note from (3.2) that $\tau(\xi(z))=0$ and the homogeneity property $\Phi(\alpha z)=\alpha^{-2} \Phi(z), \alpha>0$.

In the next Corollary, we show how the monotonicity of the free Fisher information follows easily from Theorem 1.1.

Corollary 3.1. Let $\left(x_{i}\right)_{i \in \mathbb{N}}$ be a sequence of free, identically distributed, self-adjoint random variables in $(M, \tau)$ and denote $s_{k}:=x_{1}+\ldots+x_{k}$ for every positive integer $k$. Then for all positive integers $m \leq n$, we have

$$
\Phi\left(\frac{s_{n}}{\sqrt{n}}\right) \leq \Phi\left(\frac{s_{m}}{\sqrt{m}}\right) .
$$

Proof. Assume the existence of $\xi\left(s_{1}\right)$, as otherwise $\Phi\left(s_{1}\right)=\infty$ and there is nothing to prove. According to [12, p. 206], the free sum $s_{n}=s_{m}+\left(s_{n}-s_{m}\right)$ admits $\xi\left(s_{n}\right)=$ $\operatorname{proj}_{L^{2}\left(s_{n}\right)}\left(\xi\left(s_{m}\right)\right)$ as conjugate variable. Therefore, by Theorem 1.1,

$$
\begin{aligned}
\Phi\left(s_{n}\right)=\left\|\operatorname{proj}_{\mathrm{L}^{2}\left(s_{n}\right)}\left(\xi\left(s_{m}\right)\right)\right\|_{2}^{2} & =\operatorname{cov}\left(\operatorname{proj}_{\mathrm{L}^{2}\left(s_{n}\right)}\left(\xi\left(s_{m}\right)\right), \xi\left(s_{m}\right)\right) \\
& \leq \sqrt{\frac{m}{n}}\left\|\operatorname{proj}_{\mathrm{L}^{2}\left(s_{n}\right)}\left(\xi\left(s_{m}\right)\right)\right\|_{2}\left\|\xi\left(s_{m}\right)\right\|_{2},
\end{aligned}
$$

i.e., $\Phi\left(s_{n}\right) \leq \frac{m}{n} \Phi\left(s_{m}\right)$. We conclude by the homogeneity property.

In view of (3.1) and the divisibility of the semicircular distribution w.r.t. the free convolution, the above corollary readily implies (1.1), thus proving Corollary 1.2.

\section{Monotonicity of the free Stein discrepancy}

The goal of this section is to provide a proof of Corollary 1.3. Let us fix $m \leq n$ and $x_{1}, x_{2}, \ldots$ a sequence of free, centered, identically distributed, self-adjoint random variables in $(M, \tau)$ with unit norm. Let us record the following consequence of Theorem 1.1 which will be used in the sequel.

Lemma 4.1. Let $K \in \mathrm{L}^{2}\left(s_{m}\right) \otimes \mathrm{L}^{2}\left(s_{m}\right)$ and suppose that $\langle K, 1 \otimes 1\rangle=1$. Then

$$
\left\|\operatorname{proj}_{\mathrm{L}^{2}\left(s_{n}\right) \otimes \mathrm{L}^{2}\left(s_{n}\right)}(K-1 \otimes 1)\right\|_{\mathrm{L}^{2}\left(s_{n}\right) \otimes \mathrm{L}^{2}\left(s_{n}\right)} \leq \sqrt{\frac{m}{n}}\|K-1 \otimes 1\|_{\mathrm{L}^{2}\left(s_{m}\right) \otimes \mathrm{L}^{2}\left(s_{m}\right)} .
$$

Proof. Let us denote by $Q$ the projection on 1 and write $P_{n}$ (resp. $P_{m}$ ) for $\operatorname{proj}_{\mathrm{L}^{2}\left(s_{n}\right)}$ (resp. $\left.\operatorname{proj}_{\mathrm{L}^{2}\left(s_{m}\right)}\right)$. Note that using Theorem 1.1, we have for any $b \in \mathrm{L}^{2}\left(s_{m}\right)$

$$
\left\|\left(P_{n}-Q\right)(b)\right\|_{2}^{2}=\left\langle\left(P_{n}-Q\right)(b), b-Q(b)\right\rangle \leq \sqrt{\frac{m}{n}}\left\|\left(P_{n}-Q\right)(b)\right\|_{2}\|b-Q(b)\|_{2},
$$

which leads to

$$
\left\|\left(P_{n}-Q\right)(b)\right\|_{2} \leq \sqrt{\frac{m}{n}}\|b-Q(b)\|_{2} .
$$

Using that $P_{\ell} Q=Q P_{\ell}=Q$ for $\ell \in\{n, m\}$, the above relation can be equivalently stated as

$$
P_{m} P_{n} P_{m}-Q \preceq \frac{m}{n}\left(P_{m}-Q\right),
$$

where $\preceq$ denotes the positive semi-definite ordering. Using this we can write

$$
\begin{aligned}
P_{m} P_{n} P_{m} \otimes P_{m} P_{n} P_{m}-Q \otimes Q & =P_{m} P_{n} P_{m} \otimes\left(P_{m} P_{n} P_{m}-Q\right)+\left(P_{m} P_{n} P_{m}-Q\right) \otimes Q \\
& \preceq \frac{m}{n} P_{m} \otimes\left(P_{m}-Q\right)+\frac{m}{n}\left(P_{m}-Q\right) \otimes Q \\
& =\frac{m}{n}\left(P_{m} \otimes P_{m}-Q \otimes Q\right),
\end{aligned}
$$


where we have also used that $P_{m} P_{n} P_{m} \preceq P_{m}$. Note that since $\langle K, 1 \otimes 1\rangle=1$, then $K-1 \otimes 1=\left(P_{m} \otimes P_{m}-Q \otimes Q\right)(K)$. The proof is finished after observing that

$$
\left(P_{m} \otimes P_{m}-Q \otimes Q\right) P_{n} \otimes P_{n}\left(P_{m} \otimes P_{m}-Q \otimes Q\right)=P_{m} P_{n} P_{m} \otimes P_{m} P_{n} P_{m}-Q \otimes Q,
$$

and combining it with the above.

Given $K \in \mathrm{L}^{2}\left(s_{m}\right) \otimes \mathrm{L}^{2}\left(s_{m}\right)$ a free Stein kernel of $s_{m} / \sqrt{m}$, we have by the definition of $K$ (and homogeneity) that

$$
\left\langle\frac{s_{m}}{\sqrt{m}}, P\left(s_{m}\right)\right\rangle=\sqrt{m}\left\langle K, \partial P\left(s_{m}\right)\right\rangle
$$

for every polynomial $P$. Since $s_{m}$ and $s_{n}-s_{m}$ are free, then using the intertwining relation between $\partial$ and the conditional expectation [19], we can write

$$
\left\langle\frac{s_{m}}{\sqrt{m}}, P\left(s_{n}\right)\right\rangle=\sqrt{m}\left\langle K, \partial P\left(s_{n}\right)\right\rangle \text {. }
$$

The above relation appears in [3] (before Lemma 2.5 there). Using linearity and that the $x_{i}$ 's are identically distributed, we get

$$
\left\langle s_{m}, P\left(s_{n}\right)\right\rangle=m\left\langle x_{1}, P\left(s_{n}\right)\right\rangle=\frac{m}{n}\left\langle s_{n}, P\left(s_{n}\right)\right\rangle .
$$

Putting the above relations together, we deduce that

$$
\left\langle\frac{s_{n}}{\sqrt{n}}, P\left(s_{n}\right)\right\rangle=\sqrt{n}\left\langle K, \partial P\left(s_{n}\right)\right\rangle
$$

for every polynomial $P$. This implies that $\operatorname{proj}_{\mathrm{L}^{2}\left(s_{n}\right) \otimes \mathrm{L}^{2}\left(s_{n}\right)}(K)$ is a free Stein kernel of $s_{n} / \sqrt{n}$. Thus, we have

$$
\Sigma^{*}\left(\frac{s_{n}}{\sqrt{n}} \mid S\right) \leq \inf _{K}\left\|\operatorname{proj}_{\mathrm{L}^{2}\left(s_{n}\right) \otimes \mathrm{L}^{2}\left(s_{n}\right)}(K-1 \otimes 1)\right\|_{\mathrm{L}^{2}\left(s_{n}\right) \otimes \mathrm{L}^{2}\left(s_{n}\right)},
$$

where the infimum is taken over all free Stein kernels $K \in \mathrm{L}^{2}\left(s_{m}\right) \otimes \mathrm{L}^{2}\left(s_{m}\right)$ of $s_{m} / \sqrt{m}$. Noting that $\langle K, 1 \otimes 1\rangle=1$, then using Lemma 4.1 we deduce

$$
\Sigma^{*}\left(\frac{s_{n}}{\sqrt{n}} \mid S\right) \leq \sqrt{\frac{m}{n}} \inf _{K}\|K-1 \otimes 1\|_{\mathrm{L}^{2}\left(s_{m}\right) \otimes \mathrm{L}^{2}\left(s_{m}\right)},
$$

where the infimum is again taken over all free Stein kernels $K \in \mathrm{L}^{2}\left(s_{m}\right) \otimes \mathrm{L}^{2}\left(s_{m}\right)$ of $s_{m} / \sqrt{m}$. Corollary 1.3 then follows after noting that the projection on $\mathrm{L}^{2}\left(s_{m}\right) \otimes \mathrm{L}^{2}\left(s_{m}\right)$ of every free Stein kernel of $s_{m} / \sqrt{m}$ is also a free Stein kernel.

\section{References}

[1] Shiri Artstein, Keith M. Ball, Franck Barthe, and Assaf Naor, Solution of Shannon's problem on the monotonicity of entropy, J. Amer. Math. Soc. 17 (2004), no. 4, 975-982. MR-2083473

[2] Philippe Biane, Processes with free increments, Math. Z. 227 (1998), no. 1, 143-174. MR1605393

[3] Guillaume Cébron, A quantitative fourth moment theorem in free probability theory, Adv. Math. 380 (2021), 107579. MR-4203499

[4] Guillaume Cébron, Max Fathi, and Tobias Mai, A note on existence of free Stein kernels, Proc. Amer. Math. Soc. 148 (2020), no. 4, 1583-1594. MR-4069196

[5] Thomas A. Courtade, Monotonicity of entropy and Fisher information: a quick proof via maximal correlation, Commun. Inf. Syst. 16 (2016), no. 2, 111-115. MR-3638565 
Maximal correlation and monotonicity of free entropy and of Stein discrepancy

[6] Thomas A. Courtade, Max Fathi, and Ashwin Pananjady, Existence of Stein kernels under a spectral gap, and discrepancy bounds, Ann. Inst. Henri Poincaré Probab. Stat. 55 (2019), no. 2, 777-790. MR-3949953

[7] Amir Dembo, Abram Kagan, and Lawrence A. Shepp, Remarks on the maximum correlation coefficient, Bernoulli 7 (2001), no. 2, 343-350. MR-1828509

[8] B. Efron and C. Stein, The jackknife estimate of variance, Ann. Statist. 9 (1981), no. 3, 586-596. MR-0615434

[9] Max Fathi and Brent Nelson, Free Stein kernels and an improvement of the free logarithmic Sobolev inequality, Adv. Math. 317 (2017), 193-223. MR-3682667

[10] Fumio Hiai and Dénes Petz, The semicircle law, free random variables and entropy, Mathematical Surveys and Monographs, vol. 77, American Mathematical Society, Providence, RI, 2000. MR-1746976

[11] Zhengfeng Ji, Anand Natarajan, Thomas Vidick, John Wright, and Henry Yuen, MIP*=RE, 2020. arXiv:2001.04383

[12] James A. Mingo and Roland Speicher, Free probability and random matrices, Fields Institute Monographs, vol. 35, Springer, New York; Fields Institute for Research in Mathematical Sciences, Toronto, ON, 2017. MR-3585560

[13] Alexandru Nica, Dimitri Shlyakhtenko, and Roland Speicher, Operator-valued distributions. I. Characterizations of freeness, Int. Math. Res. Not. (2002), no. 29, 1509-1538. MR-1907203

[14] Alexandru Nica and Roland Speicher, On the multiplication of free $N$-tuples of noncommutative random variables, Amer. J. Math. 118 (1996), no. 4, 799-837. MR-1400060

[15] Dimitri Shlyakhtenko, A free analogue of Shannon's problem on monotonicity of entropy, Adv. Math. 208 (2007), no. 2, 824-833. MR-2304337

[16] Dimitri Shlyakhtenko and Terence Tao. With an appendix by David Jekel, Fractional free convolution powers, 2020. arXiv:2009.01882

[17] Dan Voiculescu, The analogues of entropy and of Fisher's information measure in free probability theory. II, Invent. Math. 118 (1994), no. 3, 411-440. MR-1296352

[18] Dan Voiculescu, The analogues of entropy and of Fisher's information measure in free probability theory. V. Noncommutative Hilbert transforms, Invent. Math. 132 (1998), no. 1, 189-227. MR-1618636

[19] Dan Voiculescu, Lectures on free probability theory, Lectures on probability theory and statistics (Saint-Flour, 1998), Lecture Notes in Math., vol. 1738, Springer, Berlin, 2000, pp. 279-349. MR-1775641

[20] Dan Voiculescu, Free entropy, Bull. London Math. Soc. 34 (2002), no. 3, 257-278. MR1887698

Acknowledgments. The authors would like to thank the anonymous referees for their numerous generous suggestions which greatly improved the manuscript. For instance, the monotonicity of free Stein discrepancy and its proof was suggested by one of the referees. The authors are grateful to Roland Speicher for helpful comments and for bringing to their attention the recent preprint [16]. The second named author is thankful to Marwa Banna for helpful discussions. 